\title{
Research on Compression Method of Biomass Dense Forming Process
}

\section{Based on Discrete Element Method}

\author{
Zhen $\mathrm{Li}^{1, \mathrm{a}}$, Yuhang Gao ${ }^{1, \mathrm{~b}}$, Peng Liu ${ }^{1, \mathrm{c}}$ \\ ${ }^{1}$ College of Machine Engineering, Inner Mongolia University of Science and Technology, Baotou, \\ China \\ ${ }^{a}$ lizhen730106@126.com, ${ }^{\mathrm{b}}$ 1271746279@qq.com, ㄷ1578948@qq.com
}

Keywords: discrete element, sandy shrub, compression method, forming quality, energy consumption

Abstract. The purpose of this research is to analyze the molding quality and energy consumption of biomass pellet fuel under three compression methods of uniform speed, uniform deceleration and superposition vibration. This article established a discrete element model of sandy shrub particles uniaxial compressed. The simulation results shows that the compression method has no significant influence on the maximum compression force. The degree of particle aggregation and deformation is better than the other methods in vibration compression method. The forming quality of vibration compression method is better than the other methods. The particle bonding effect is poor in uniform compression method. This research calculates the work of uniform speed, uniform deceleration and superposition vibration by using numerical integration through the force-displacement data. They are respectively $25.898 \mathrm{~J}, 24.496 \mathrm{~J}, 28.019 \mathrm{~J}$. This result shows that vibration compression method consumes the most energy, the uniform deceleration compression method is the least. The total energy of particles under different compression methods can also indicate the accuracy of this conclusion. The simulation results can provide a theoretical basis for the selection of compression methods in the process of biomass solidification.

\section{Introduction}

The development of new energy is imminent. It's closely watched by countries all over the world due to emphasizing on environmental protection and future development of human society. Biomass energy is a green and non-polluting renewable energy compared to other kinds of energy $\mathrm{y}^{[1]}$. Sandy shrub resources are very rich in China. Its compact compression treatment can provide a way for the effective utilization of vast sandy resources. It is also important to reduce environmental pollution and ease energy shortage.

In recent years, domestic and foreign scholars had carried out a lot of researches on biomass dense molding technology, and obtained a number of research results, which provide a certain research foundation for the research of high-efficiency and energy-saving compact forming technology. Xiaojie Zhou ${ }^{[2]}$ test axial and radial compression forces of the clover by using a special compression test device. It is found that there is a linear relationship between axial and radial compression forces. The compression velocity and initial density have a great influence on the radial compression force when the compression density is more than $50 \mathrm{~kg} / \mathrm{m}^{3}$. Mingshao Yang ${ }^{[3]}$ study the rheological properties of grass materials by open compression tests, and conclude that the movement of grass blades is an iterative process of expansion and compression. Rolfe ${ }^{[4]}$ studies $^{4}$ shows that the increase of ring velocity makes the material height decrease, thus the extrution force 
is decreases, it results the decrease of the molding quality. And the increase of particles temperature results the increase of energy consumption. Xinzhu Liu ${ }^{[5]}$ conduct a closed compression test of different compression velocity for the same type and different lengths straws on an electronic universal material testing machine. The results show that the greater compression velocity, the greater pressure under the same compression density. Yuezhu Sun ${ }^{[6]}$ research the compression and stress relaxation process of corn straws unidirectional compressed at different compression speeds. The results show that the change of compression speed has little effect on the balance force after stress relaxation when the compression displacement is constant. The above studies focus more on the effect of compression speed on compression force and the molding quality, and does not study the movement method of loading surface during compression.

Because of cell structure and arrangement and so on of biomass materials, its mechanical properties are mostly anisotropic ${ }^{[7]}$. Particles are a discrete state before formation, and it is a acicular geometry. It is difficult to study exactly with the continuous physical analysis method, so it is more practical to adopt the discrete element method. Impact and dwell time are the main factors affecting compacting process of biomass. This paper simulates the extrusion process of uniform speed, uniform deceleration and superposition vibration taking sandy shrub particles as the research object by using discrete element method. The research analyzes the molding quality and energy consumption of forming fuels under three compression methods.

\section{Theoretical basis}

Discrete Element Method (DEM) is a particle material micromechanical analysis method proposed by Cundall in 1971 based on the interaction between elements and Newton's law of motion ${ }^{[8]}$. The solution process is cumbersome in discrete element spherical particle contact theory. It is necessary to simplify the process without producing significant errors. At present, the simplified models mainly includes soft ball model and hard ball model. The hard ball model ignores the surface deformation of particles, and it's mainly applied to the numerical simulation of rapid collisions between particles with low concentration. The soft ball model calculates the contact force based on the normal overlap and tangential displacement between particles. It is suitable for numerical calculation of engineering problems due to the low computational intensity ${ }^{[9]}$.

The particle collision can produce a significant plastic deformation because sandy shrubs are a viscoelastic material. Therefore, the soft ball model (Fig. 1) is used to describe the contact between particles. This model simplifies the normal contact mechanics model into spring $\left(K_{\mathrm{n}}\right)$ and damper $\left(\eta_{\mathrm{n}}\right)$. The tangential contact mechanics model is simplified to spring $\left(K_{\mathrm{s}}\right)$, damper $\left(\eta_{\mathrm{s}}\right)$ and sliding friction gear $(\mu)$. The coupler $(C)$ is used to determine the particle-pairing relationship when the contact occurred. The force between particles surface is very weak compared to the contact force. The particles are homogeneous in the macroscopic field and the contact surface is small compared to the particles surface. Therefore, the Hertz-Mindlin (no slip) contact model is chosen to simulate the extrusion process. 


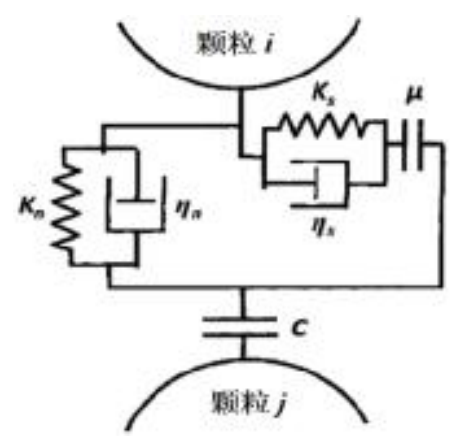

Particles $i, j$ are in contact; $K_{\mathrm{n}}$ : Normal stiffness, $K_{\mathrm{s}}$ : Shear stiffness $(\mathrm{N} / \mathrm{m}) ; \eta_{\mathrm{n}}$ : Normal damping, $\eta_{\mathrm{s}}$ : Shear damping; $\mu$ : Friction between particles; $C$ : Relationship between particles

Fig.1 Soft ball model

\section{Discrete element simulation}

Discrete element model. In order to promote sandy shrub particles to enter the cavity more easily, there should be a certain taper at the feed inlet. The size of the taper is related to the force of the conical surface. The stress is small on the feed cone when the taper angle is $60^{\circ}{ }^{\circ 11]}$. So, the mold of $60^{\circ}$ cone angle is selected. The Length to diameter ratio of molding cavity is selected as 6:1 through literature research ${ }^{[12]}$. The dimension parameters of the mold shown in Fig.2a. The discrete element model built from mold parameters and constraint conditions in Fig.2a shown in Fig.2b.

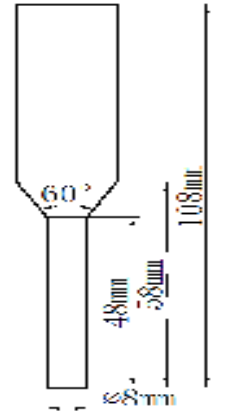

a. Mold parameters

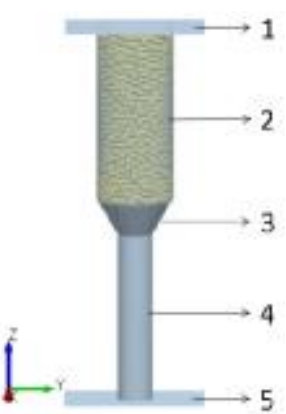

b. Discrete element model

1.Load surface 2.Storage bin 3.Feed cone 4.Mold cavity 5.Bottom baffle

Fig.2 Mold parameters and discrete element model

Particle model and contact parameters. Sandy shrub particles size range is $0.5 \sim 1.5 \mathrm{~mm}$ after sieving in molding process. Therefore, this simulation uses spherical particles with a mean radius of $1 \mathrm{~mm}$ to replace the original material. Table 1 shows the material parameters ${ }^{[13]}$. Table 2 shows the contact parameters $^{[14]}$.

Tab.1 Material parameters

\begin{tabular}{lccccc}
\hline Items & $\begin{array}{c}\text { Poisson' } \\
\text { Ratio }\end{array}$ & $\begin{array}{c}\text { Shear } \\
\text { Modulus[Pa }]\end{array}$ & $\begin{array}{c}\text { Density } \\
{\left[\mathrm{Kg} \cdot \mathrm{m}^{-3}\right]}\end{array}$ & $\begin{array}{c}\text { Normal } \\
\text { stiffness }\left[\mathrm{N} \cdot \mathrm{m}^{-1}\right]\end{array}$ & $\begin{array}{c}\text { Shear } \\
\text { stiffness }\left[\mathrm{N} \cdot \mathrm{m}^{-1}\right]\end{array}$ \\
\hline Sandy shrubs & 0.4 & $2 \times 10^{8}$ & 1500 & $6.2 \times 10^{5}$ & $2.43 \times 10^{5}$ \\
Mold & 0.3 & $7 \times 10^{10}$ & 7800 & $10^{8}$ & $10^{8}$ \\
\hline \multicolumn{5}{c}{ Tab.2 } & Contact parameters \\
\hline \multirow{2}{*}{ Items } & Coefficient of & Coefficient of Static & Coefficient of Rolling \\
& Restitution & \multicolumn{2}{c}{ Friction } & Friction \\
\hline Sandy shrubs-Sandy shrubs & 0.5 & 0.5 & 0.02 \\
Sandy shrubs -Mold & 0.7 & 0.65 & 0.03 \\
\hline
\end{tabular}

Setting working parameters and particle factory. Particle factory randomly generates 
particles according to the cavity volume and particle size range. The mold cavity filled with particles. It is equivalent to the initial state of compression after the material is filled in the experiment. The loading surface squeezes particles by uniform speed, uniform deceleration and superposition vibration. The purpose of using uniform deceleration compression method is to increase the dwell time. The purpose of using superposition vibration compression method is to provide impact so that particles are compacted more densely.

The gravity acceleration is $9.81 \mathrm{~m} / \mathrm{s}^{2}$, and its direction is Z-axis negative direction. Particle factory uses a dynamic method to generate 2500 particles at a rate of 1000/s. Taking $29 \%$ of the Rayleigh time step as a fixed time step, which is $2.649 \times 10^{-6} \mathrm{~s}$. The total simulation time is $50 \mathrm{~s}$. The total movement displacement is $50 \mathrm{~mm}$. Setting the cell size to $3 \mathrm{Rmin}$ (minimum particle radius). The moving velocity of uniform speed is $1 \mathrm{~mm} / \mathrm{s}$, and its direction is Z-axis negative direction. The initial velocity of uniform deceleration is $2 \mathrm{~mm} / \mathrm{s}$, and its direction is $\mathrm{Z}$-axis negative direction. The acceleration is $0.04 \mathrm{~mm} / \mathrm{s}^{2}$, and its direction is Z-axis positive direction. The uniform velocity of superposition vibration is $1 \mathrm{~mm} / \mathrm{s}$, and its direction is Z-axis negative direction. The vibration amplitude is $0.5 \mathrm{~mm}$, and the frequency is $5 \mathrm{~Hz}$.

\section{Results and discussion}

Compression force analysis. Compression force-time curve shown in Fig.3. The comparative analysis shows that the compression methods has little effect on the maximum compression force, but has a significant effect on dwell time and force variation trend.

The dwell time is longer in uniform deceleration method. The external load increases the time that particles bond together. It is more difficult for deformed particles to restore the original state. Therefore, the molding fuel quality is better than uniform speed method.

The compression force-time curve is basically coincide by comparing uniform speed and superposition vibration, but the curve of vibration has fluctuating tendency. Vibration can not only provide impact to materials, but also reduce resistance in the compression process, prevent the recovery of deformed particles and improve the quality of molding fuel.

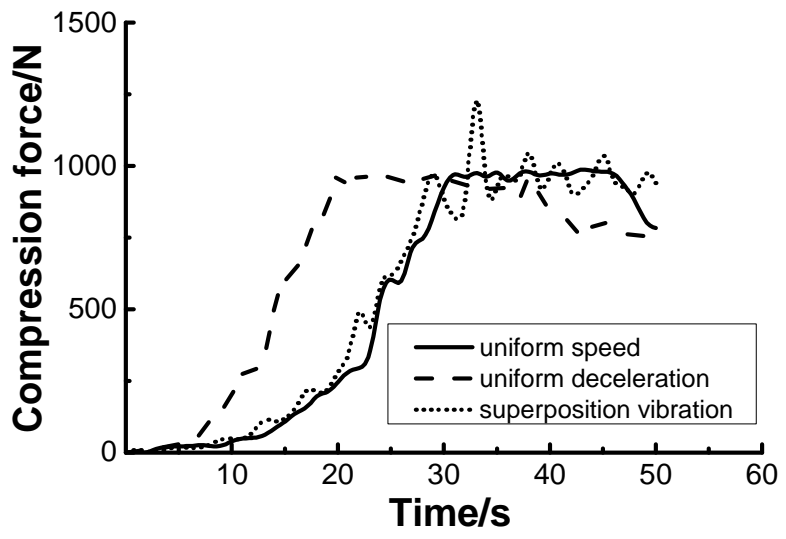

Fig.3 Compression force-time curve under different compression methods

Particle contact quantity analysis. Particle contact quantity under three compression methods shown in Fig.4. The trend of the three curves is roughly same, but three is a certain difference in the maximum contact quantity. The maximum contact quantity is 14278 in superposition vibration compression method, which is higher than the other compression methods. As the maximum contact quantity represents the degree of particle agglomeration, so the degree is greatest under vibration compression method. Its molding fuel quality is better than the other compression method. The 
molding quality of uniform deceleration is better compared to uniform method.

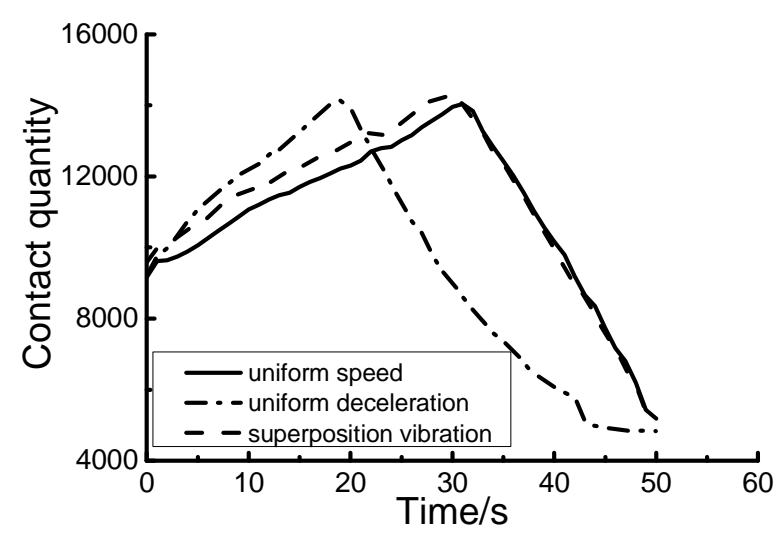

Fig.4 Particle contact quantity curve

Particle contact vector analysis. Particle contact vector curve shown in Fig.5. The contact vector describes the distance between centers of any two contact particles. The particle deformation of vibration method is the largest, uniform deceleration method is the second, and uniform method is the least when the compression reaches a steady state. The forming quality of superposition vibration is better than the other methods according to the degree of particle deformation.

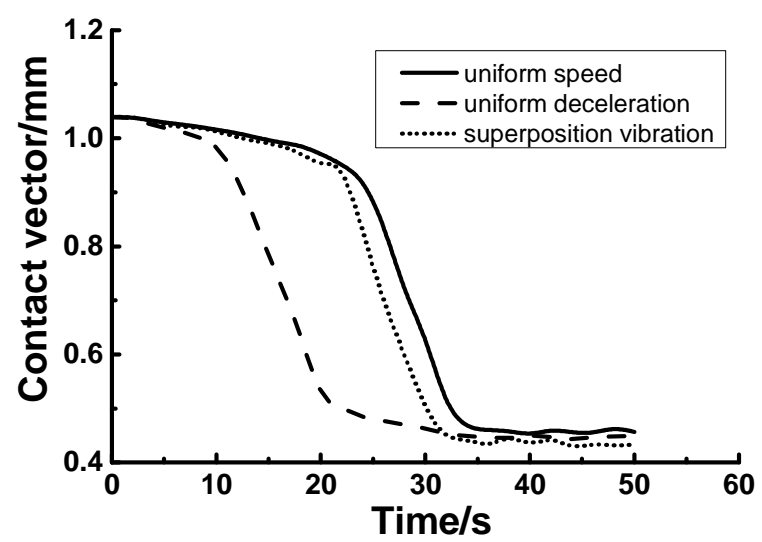

Fig.5 Particle contact vector curve

Energy analysis. Force-displacement curve shown in Fig.6 under three compression methods. The numerical integration is used to calculate the work of three compression methods through force-displacement date by using origin software. Uniform speed method is $25.898 \mathrm{~J}$, uniform deceleration method is $24.496 \mathrm{~J}$, superposition vibration method is $28.019 \mathrm{~J}$.

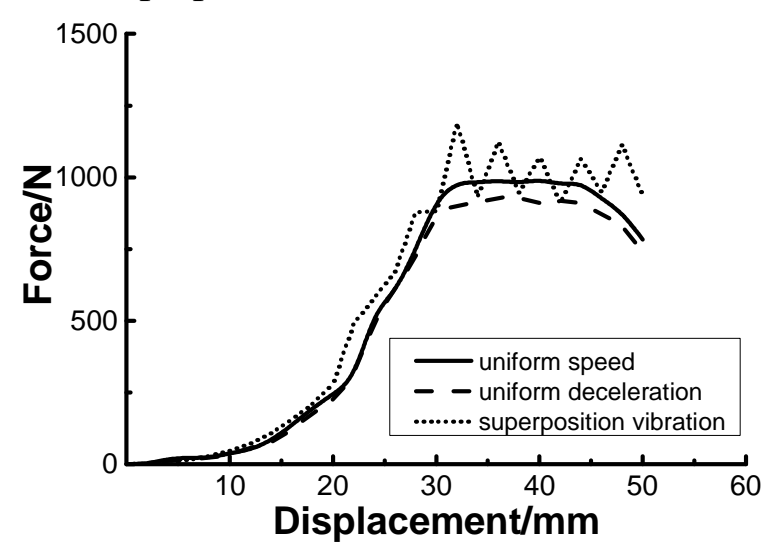

Fig.6 Force-displacement curve

The total energy of particles shown in Fig.7. It can be seen from the figure that the total energy of superposition vibration method is higher than the other two compression methods. Uniform deceleration method has the lowest energy. 


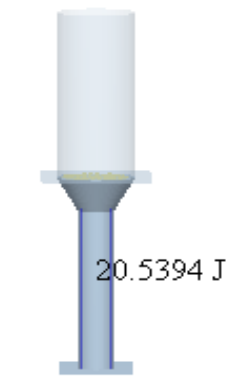

a. Uniform speed

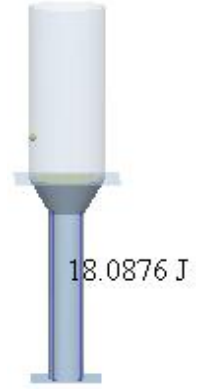

b.Uniform deceleration

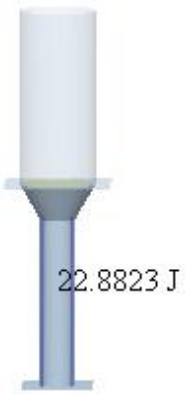

c.Superposition vibration

Fig.7 The total energy of particles

\section{Conclusions}

(1) This paper established a discrete element model of sandy shrub particles uniaxial compressed to analyze the molding quality and energy consumption under three compression methods of uniform speed, uniform deceleration, and superposition vibration. The simulation result shows that the compression methods has no significant effect on the maximum compression force.

(2) The degree of particle aggregation and deformation is better than the other methods under superposition vibration method by analyzing contact quantity and contact vector between particles. Its forming quality is better. The degree of particle aggregation and deformation is the lowest under uniform speed method. Its forming quality is poor.

(3) By analyzing the work of three compression methods, it is concluded that superposition vibration method consumes the most energy, uniform speed method is the second, uniform deceleration method is the least. The total energy of particles under different compression methods also shows the accuracy of this conclusion.

The article analyzes the molding quality and energy consumption under three compression methods. The simulation results provide a reference for the selection of compression method in the biomass pellet fuel compacting process.

\section{Acknowledgements}

This work was financially supported by the Natural Science Foundation of China (51666016).

\section{References}

[1] H.R. Liu, G.B. Liu, L.J. Li, et al: Journal of Forestry Engineering Vol. 1 (2016), p. 93

[2] X.J. Zhou, C.G. Wang: Transactions of the Chinese Society for Agriculture Machinery Vol. 40 (2009), p. 115

[3] M.S. Yang, X.Y. Li: Journal of Agricultural Mechanization Research Vol. 27 (2005), p. 81

[4] L.A. Rolfe, H.E. Huff, F. Hsiech: Journal of Aquatic Food Product Technology Vol. 10 (2001), p. 21

[5] X.Z. Liu, Y.H. Wang, P. Han, et al: Journal of Agricultural Mechanization Research Vol. 38 (2016), p. 225

[6] Y.Z. Sun: Design, Research on Briquetting Mechanism of Extruding Corn Stalk Powder Based on Discrete Element Method (M.S. Shenyang Agricultural University, China 2016), p. 37

[7] Q.X. Sun, R.J. Zhang, Y.P. Dong: Transactions of the Chinese Society for Agriculture Machinery Vol. 40 (2009), p. 130 
[8] P.A. Cundall: International Journal of Rock Mechanics and Mining Sciences and Geo-mechanics Abstracts Vol. 25 (1988), p.107

[9] Y.k. Li, Y.Z. Sun, X.W. Bai: Transactions of the Chinese Society of Agricultural Engineering Vol. 31 (2015), p.212

[10] F.G. Jia, L.N. Yao, Y.L. Han, et al: Transactions of the Chinese Society of Agricultural Engineering Vol. 32 (2016), p. 54

[11] Z. Li, B. Xue, G.S. Yu: Feed Industry Vol. 35 (2014), p. 23

[12] X.F. He, T.Z. Lei, Z.F. Li, et al: Acta Energiae Solaris Sinica Vol. 27 (2006), p. 937

[13] F.Y. Liu, J. Zhang, B. Li, et al: Transactions of the Chinese Society of Agricultural Engineering Vol. 32 (2016), p. 247

[14] T. Zhang, F. Liu, M.Q. Zhao, et al: Transactions of the Chinese Society of Agricultural Engineering Vol.32 (2016), p.27 